\title{
Photonics tools begin to clarify astrocyte calcium transients
}

\author{
Kelsea A. Gorzo and Grant R. Gordon $\odot^{*}$ \\ University of Calgary, Hotchkiss Brain Institute, Cumming School of Medicine, Calgary, \\ Alberta, Canada
}

\begin{abstract}
Astrocytes integrate information from neurons and the microvasculature to coordinate brain activity and metabolism. Using a variety of calcium-dependent cellular mechanisms, these cells impact numerous aspects of neurophysiology in health and disease. Astrocyte calcium signaling is highly diverse, with complex spatiotemporal features. Here, we review astrocyte calcium dynamics and the optical imaging tools used to measure and analyze these events. We briefly cover historical calcium measurements, followed by our current understanding of how calcium transients relate to the structure of astrocytes. We then explore newer photonics tools including super-resolution techniques and genetically encoded calcium indicators targeted to specific cellular compartments and how these have been applied to astrocyte biology. Finally, we provide a brief overview of analysis software used to accurately quantify the data and ultimately aid in our interpretation of the various functions of astrocyte calcium transients. $\odot$ The Authors. Published by SPIE under a Creative Commons Attribution 4.0 International License. Distribution or reproduction of this work in whole or in part requires full attribution of the original publication, including its DOI. [DOI: 10.1117/1.NPh.9.2.021907]
\end{abstract}

Keywords: astrocyte; calcium; stimulation emission depletion; genetically encoded fluorescent calcium indicator; two-photon; analysis.

Paper 21064SSVR received Nov. 8, 2021; accepted for publication Feb. 1, 2022; published online Feb. 18, 2022.

\section{Introduction}

The past 30 years have witnessed a dramatic reappraisal of the role astrocytes play in the brain. With a central position in the neurovascular unit, each astrocyte is tasked with integrating information from both neurons and the microvasculature to coordinate brain activity and metabolism. As such, new compelling evidence demonstrates previously unimagined roles for these cells in many aspects of neuro(patho)physiology. Many investigators are recognizing the importance of studying astrocytes and the diverse effects these cells exert on synapses, mural cells, endothelial cells, and other glial targets. ${ }^{1-3}$ It is believed that a primary mechanism through which astrocytes transduce and encode information is by elevations in intracellular calcium. ${ }^{4}$ These calcium events are incredibly diverse, varying in area, amplitude, duration, propagation direction, and compartmental localization. Furthermore, calcium transients can be evoked by various physiological changes, synaptic activity, pathological events, or simply occur spontaneously. ${ }^{5}$ These calcium signals occur throughout different compartments of the cell. Astrocytes have complex morphology in which the majority of the cell volume (up to $85 \%$ ) ${ }^{6,7}$ is comprised of irregular, nanoscopic $\left(30\right.$ to $50 \mathrm{~nm}$ ) processes that sit below the diffraction limit of light. ${ }^{8}$ As such, the implementation of new microscopy and labeling approaches has been necessary to tease apart different calcium sources. Understanding how changes in astrocyte calcium drive communication at the neuronal-glial-vascular interface is a vibrant area of research; however, its success is closely linked to the availability and efficacy of fluorescent indicators, optical imaging methods, and analysis tools.

In this review, we cover measurements of calcium dynamics in astrocytes using optical imaging tools. First, we briefly describe how calcium signaling emerged as an important player in neuronal-astrocyte communication and glial-vascular communication, followed by an outline of our current understanding of calcium transients in relationship to the structure of astrocytes.

*Address all correspondence to Grant R. Gordon, gordong@ucalgary.ca 
Then, we delve into the tools used to accomplish this, spanning from the development of calcium-specific, small molecule dyes up to the current use of genetically encoded calcium indicators (GECIs). We then review microscopy methods particularly well suited to astrocyte calcium imaging and conclude with describing the latest analysis software, which will be vital to decoding the biological relevance of calcium transients in astrocytes.

\section{Importance of Astrocyte Calcium Emerges}

An important clue pointing to previously undiscovered roles of astrocytes in neurophysiology came with the finding that astrocytes express glutamate-sensitive ion channels. ${ }^{9}$ Following this, the seminal discovery of Cornell-Bell et al. ${ }^{10}$ demonstrated that bath application of glutamate induces calcium waves in the cytoplasm of astrocytes introducing the potential link between neural activity and calcium transients. This phenomenon was later confirmed using stimulation protocols in slice ${ }^{11,12}$ and then later in vivo via sensory stimulation in awake animals. ${ }^{13}$ Following this, the next step in recognizing fluctuations in astrocyte calcium as an extra neuronal signaling system came with the discovery that these changes regulate the release of key molecules such as ATP, ${ }^{14}$ lactate, ${ }^{15}$ or hormones such as atrial natriuretic peptide. ${ }^{16}$ These effectors exert powerful changes not only on neurons to modulate synaptic transmission ${ }^{17}$ and plasticity ${ }^{18}$ but also influence other targets including the mural cells (smooth muscle cells and pericytes) of vasculature to control the diameter of cerebral blood vessels and thereby blood flow. ${ }^{19-22}$

\section{Complexities of Astrocyte Morphology}

The unique star-like morphology of astrocytes allows the many processes of these cells to come in close proximity to important targets, such as the synapse or microvasculature. ${ }^{23}$ Depending on the physical location in the astrocyte, distinct arrays of ion channels, organelles, and cellular machinery are present allowing for specialized and compartmentalized functions. ${ }^{5}$ Commonly referred to as a microdomain, these spatially restricted areas where calcium transients occur were first discovered in Bergmann glia. ${ }^{24}$ Typically, calcium microdomains describe events restricted to the ultrafine processes of the astrocyte; however, common nomenclature precisely describing the structures in which a microdomain exist is somewhat absent. Most recently, Verkhratsky and Semyanov suggest a classification system based on morphological properties of the astrocyte arbor where branches and branchlets describe the main astrocytes processes originating from the soma (resolvable with diffraction limited optical microscopy), which then split into higher order branchlets or fine leaflets, the latter of which are below the diffraction limit, thereby unresolvable with traditional microscopes. ${ }^{25}$ Other terms such as perisynaptic astrocyte processes (PAPs) ${ }^{6}$ along with nodes and shafts ${ }^{26}$ have been used to describe the nanoscopic processes of astrocytes. These are all separate from a major process arising from the soma, at least one of which becomes a specialized endfoot covering a nearby blood vessel. Endfeet come in a variety of shapes, thicknesses, and sizes, partially reflecting whether the endfoot is covering an arteriole, venule, or capillary. ${ }^{27}$

Distinguishing the structural complexity of astrocytes particularly in the context of localizing calcium transients in fine processes requires innovative imaging approaches. ${ }^{7}$ Three-dimensional (3D) reconstructions of tissue sections using electron microscopy (EM) has provided intricate detail of PAP ${ }^{28-31}$ and endfeet. ${ }^{27,32,33}$ However, a significant limitation of this approach is that fixation of the tissue is required prior to imaging, thereby limiting experimental flexibility. To capture the dynamic nature of PAPs and endfeet in situ or in vivo, recent studies have looked to superresolution microscopy methods, which are compatible with live tissue samples, such as two photon stimulation emission depletion (2P-STED). Tools such as 2P-STED provide us with the ability to examine the interactions between nanostructures under physiologically relevant states.

\section{Sources of Astrocyte Calcium}

Teasing apart the physiological role of astrocyte calcium signaling is highly dependent on the physical location in which the calcium events are occurring, the membrane protein mediating the 
calcium flux and the downstream pathways. The soma, branches, and leaflets all possess unique cellular machinery that can generate and respond to calcium elevations, which are often completely dissociated from fluctuations occurring simultaneously in other parts of the same cell. ${ }^{5}$ Typically, calcium transients in the astrocyte arbor are more frequent and appear to be produced by a wider variety of cellular components. Given a very high surface area-to-volume ratio, it was previously believed that no organelles were present in the cytosol of PAPs. However, serial EM revealed that $40 \%$ of cortical PAPs have at least one organelle (endoplasmic reticulum [ER] or mitochondria). ${ }^{34}$ Deletion of the ER $\mathrm{IP}_{3}$ receptor type $2\left(\mathrm{IP}_{3} \mathrm{R} 2\right)$, the common effector for $\mathrm{IP}_{3}$ signaling initiated by G-protein coupled receptors activity in astrocytes, ${ }^{35}$ results in a significant reduction $(\sim 60 \%)$ in the frequency and amplitude of spontaneous calcium events in the astrocyte arbor. ${ }^{36-40}$ It is important to note that $\mathrm{IP}_{3} \mathrm{Rs}$ are also found in specialized astrocyte endfeet that encase cerebral arterioles and may play an important role in coordinating cerebral blood flow with the metabolic demand of neurons. $\mathrm{IP}_{3}$ released from perisynaptic processes can initiate an $\mathrm{IP}_{3} \mathrm{R}$-dependent calcium wave that propagates through the astrocyte to the endfoot, where the increase in calcium initiates the release of vasoactive molecules, causing vasodilation. ${ }^{41}$ In terms of detecting neuronal activity, metabotropic glutamate receptors (mGluRs) on astrocytes, commonly mGluR5, coupled to $\mathrm{G}_{\mathrm{q} / 11}$ proteins stimulate $\mathrm{IP}_{3}$ production. ${ }^{42,43}$ However, variations in the presence of mGluRs in the young versus old brain have been demonstrated. Sun et al. ${ }^{44}$ report mGluR5 expression significantly decreases with age, to such a point where pharmacological activation fails to produce somatic $\mathrm{IP}_{3}$-mediated calcium increases. Other studies show calcium elevations in mature astrocytes via whisker stimulation are mediated through synaptic glutamate release, resulting in astrocyte mGluR5 activity $^{13}$ that is localized to PAPs. ${ }^{17,45}$ A plausible explanation for this discrepancy could be attributed to the focus of Sun et al. upon somatic calcium responses, which likely differ from those in the processes of astrocytes. Furthermore, as the brain matures and refinement of synaptic circuitry takes place, it is possible that confinement in the distribution of mGluRs occurs to PAPs, where they are required for localized modulation at the tripartite synapse. ${ }^{1,46}$ Other than glutamate, numerous $\mathrm{G}$ protein coupled receptors such as $\mathrm{G}_{i}$ protein $\mathrm{GABA}_{B}$ receptors, $\mathrm{G}_{i}$ protein $\mathrm{CB} 1$ receptors, or $\mathrm{G}_{q}$ protein alpha adrenergic receptors can also cause increases in calcium via convergence on an $\mathrm{IP}_{3} \mathrm{R}$ signaling pathway. ${ }^{47-51}$

Although calcium transients rely in part on release from intracellular stores, influx of calcium through transmembrane channels or transporters is not to be overlooked. ${ }^{52}$ Neurotransmitter transporters such as GLT-1 or GAT-3 can generate increases in intracellular sodium during cotransport, which ultimately reverses the activity of the $\mathrm{Na}^{+} / \mathrm{Ca}^{2+}$ exchanger (NCX), resulting in calcium influx. ${ }^{53-55}$ This effect was demonstrated with bath application of GABA. ${ }^{56}$ It also has been observed that TRPA1 generates frequent calcium influxes, localized close to the plasma membrane, but this observation has been contested. ${ }^{52}$ Shigetomi et al. ${ }^{57}$ do however, provide evidence that TRPA1 in maintains basal calcium levels and regulates d-serine release into the extracellular space. Similarly, TRPC1, another commonly expressed cation channel in astrocytes, has been shown to be responsible for replenishing internal calcium stores, in addition to driving calcium-dependent glutamate release. ${ }^{58}$

Other interesting possible sources of transmembrane calcium transients occur upon activation of mechanosensitive channels. Positioned around cerebral arterioles, astrocyte endfeet acts as intracranial baroreceptors that sense changes in perfusion via fluctuations in the vascular lumen diameter. ${ }^{59-61}$ This mechanosensory signaling is thought to be mediated through pannexin 1 (Panx1) hemichannels, ${ }^{62}$ PIEZOs, ${ }^{63}$ and TRPV4. ${ }^{61,64}$ Turovsky et al. ${ }^{64}$ demonstrated that TRPV4 in particular contributes to mechanical gating of $\mathrm{Cx} 43$ hemichannels, resulting in the release of ATP, which amplifies calcium signaling via P2Y1 receptors and recruitment of intracellular calcium stores. Haidey et al. observed that arteriole vasoconstriction elevates endfoot calcium via TRPV4, which immediately engages a feedback dilation pathway to constrain the extent of constriction. In vivo, this pathway limited the amplitude of rhythmic fluctuations in arteriole diameter. $^{61}$

Recent reports have also connected mechanical changes of PAPs to calcium signaling in response to LTP induction. PAPs, which are positioned adjacent to synapses and rich in GLT-1 transporters, are responsible for containing glutamate to the synaptic cleft. If substantial "spillover" occurs, there can be significant physiological impacts including NMDA receptormediated intersynaptic crosstalk, heterosynaptic potentiation, and remote activation of 
mGluRs. Rusakov's team demonstrate using super resolution imaging that PAPs withdraw from potentiated synapses following LTP. ${ }^{65}$ Interestingly, while this group believes this process to be mediated by NKCC1, others have found astrocytic calcium signaling more central to this process. Jones et al. overexpressed mGluR1/5, which upon stimulation increased PAP coverage of synapses and directly affected the long-term survival of these spines. ${ }^{66}$ Given that PAPs contain numerous actin-related proteins, ${ }^{45}$ Molotkov et al ${ }^{67}$ found that calcium-dependent actin binding protein, profilin-1 is essential to PAP plasticity. Further research is required to functionally link synaptic activity with modulation of the cytoskeleton. Nevertheless, the structural changes of PAPs surely influence calcium dynamics, likely impacting many important downstream processes.

\section{Calcium Indicators}

Significant advancements in the biotechnology underpinning fluorescent calcium indicators continue to drive our understanding of the complex role calcium plays in neurophysiology. The ability to measure intracellular calcium originates with small molecule dyes that consisted of a calcium chelating complex conjugated to a fluorescent reporter ${ }^{68,69}$ Fura-2, which was one of the first widely utilized indicators, demonstrates a shift in excitation spectra in the presence of calcium therefore the ratio of fluorescence emitted at two different excitation wavelengths can be used to determine the concentration of calcium. ${ }^{70}$ More recently, studies investigating rapid calcium transients in astrocytes have used bulk application of membrane permeable (acetoxymethyl (AM) esters), single wavelength calcium indicators such as Rhod-2- AM or Oregon Green BAPTA-1-AM (OGB1-AM). Rhod-2 is bright and exhibits a large change in fluorescence (100-fold or more) upon calcium binding with a Kd of $\sim 570 \mathrm{nM}$ (medium affinity). ${ }^{70}$ However, Rhod-2 also loads mitochondria, leading to mixed cell compartment calcium signals. OGB-1 is bright and has a high affinity for calcium $(\mathrm{Kd} \sim 190 \mathrm{nM})$, which is ideal to detect small concentration changes, yet has a much smaller dynamic range compared to Rhod-2 (maximum 14 -fold $)^{70,71}$ (Fig. 1). The AM versions of these dyes load astrocytes very effectively, and neither display a significant shift in the excitation/emission spectra when binding calcium. ${ }^{70}$ Relative calcium measurements are performed by calculating the ratio of fluorescence during a calcium signal over the average baseline fluorescence. This technique is simple and allows comparisons across experiments that exhibit differences in dye loading or photobleaching. An important consideration for calcium imaging in astrocytes using AM dyes, however, is primarily soma and larger processes labeling occurs, leaving PAP calcium transient detection suboptimal and poorly resolved. ${ }^{72-74}$ To combat this, nonmembrane-permeable organic calcium dyes including Fluo-4, Fura-2, or calcium green-1 potassium salts can be directly loaded into an astrocyte via whole cell patch clamp. Fluo-4 salt is commonly used because it has relatively high affinity for calcium $(\sim 340 \mathrm{nM})$, is very bright, and has a large dynamic range (>100-fold). ${ }^{73}$ Advantages of this method are improved loading of the distal fine processes and that the intracellular concentration of the dye is known. ${ }^{75}$ It may be important to perfuse a gap junction blocker such as carbenoxolone prior to loading to prevent diffusion and dilution of the dye throughout the greater astrocyte syncytium or use a calcium indicator conjugated to dextran chains to increase the molecular weight, also preventing gap junction transport. ${ }^{52,61,76}$

Nevertheless, both bulk and patch loading of these calcium indicators requires coloading a secondary fluorescent probe to identify astrocytes. Typically, the dye sulforhodamine 101 (SR101) is selected for its preferential uptake by astrocytes and forgiving loading conditions. The deep-red emission of SR101 can be detected with minimal spectral overlap with GFP or green fluorescein-based calcium indicators. ${ }^{77}$ SR101 uptake is animal age-dependent, however, ${ }^{78}$ and is not equivalent in all brain regions. ${ }^{79}$ Some studies also show at concentrations somewhat higher than that required to label astrocytes, SR101 can cause increased neuronal excitability and long-term potentiation, ${ }^{80,81}$ which is an important consideration if conducting functional studies. A popular alternative approach is to express enhanced green or yellow fluorescent protein (eGFP eYFP) or red fluorescent protein such as mCherry or tdTomato in specific cellular compartments or cell types using either using AAV or a transgenic mouse line. Common astrocyte-specific promoters used are GFAP, GfaABC1D, ALDH1L1, S100 $\beta$, glutamine synthetase, GLAST, and 


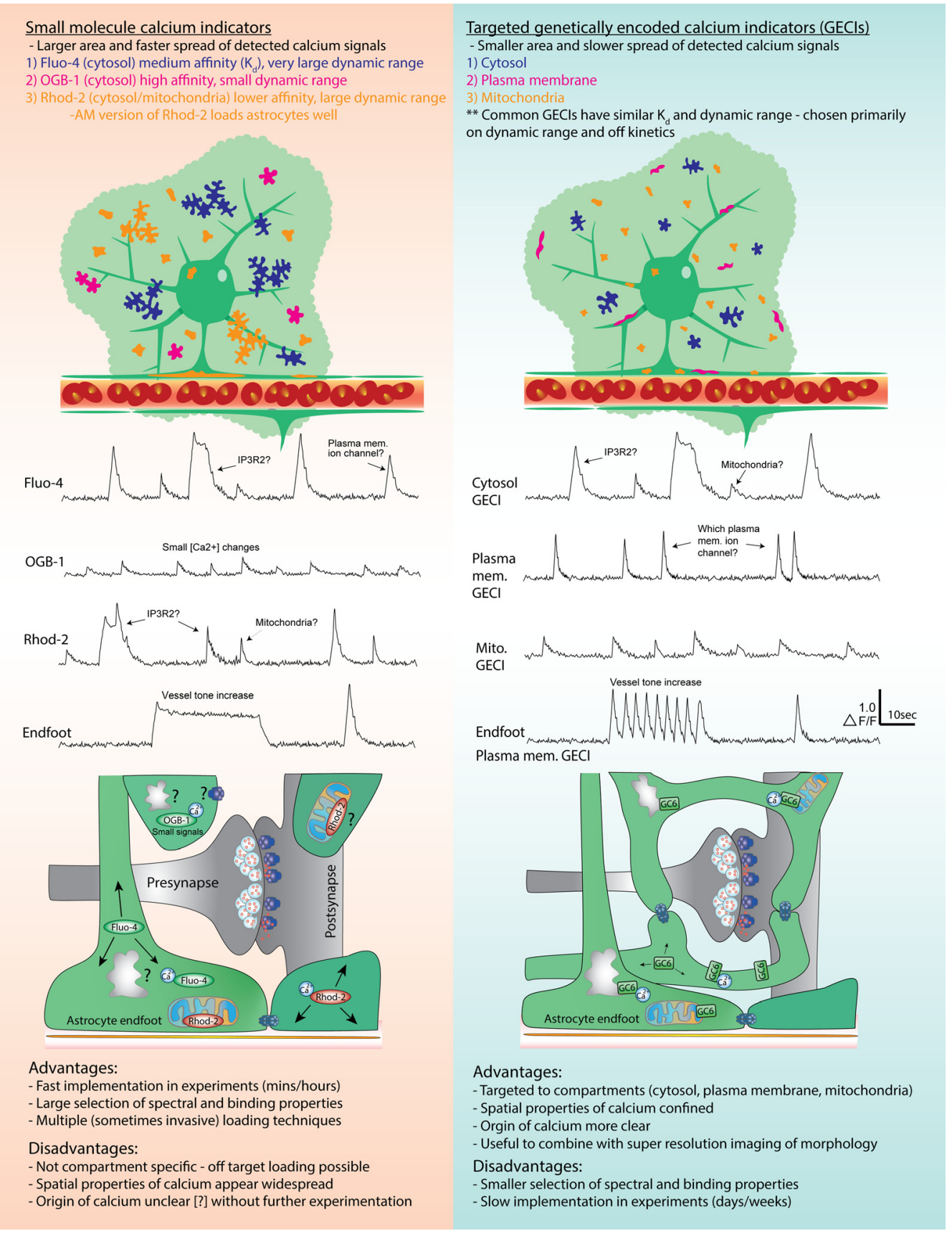

Fig. 1 A comparison of small molecule calcium indicators with GECls. A visual representation of the area of detected calcium signals inside the astrocyte is shown along with representative $\Delta F / F$ traces and general properties for various common indicators. A description of the advantages and disadvantages for small molecule indicators versus GECls is also provided.

GLT-1. ${ }^{82-86}$ However, even when a small molecule calcium indicator is combined with a cytosolic fluorescent protein of a different color to mark morphology, views of the fine astrocyte arbor are still inadequate for determining the precise location of calcium signals (see Fig. 1). This is where the development of genetically encoded fluorescent calcium indicators (GECIs) and improved optical imaging techniques have begun to enable new observations and bring insights to astrocyte calcium signaling.

The past decade has seen significant advances in the development of GECIs. GECI expression in astrocytes is now routinely implemented from the culture dish to in vivo. This technology overcomes the previous requirement of loading exogenous probes and prevents any indiscriminate 
fluorescence labeling in tissues, cells, and even organelles that are not of interest. Indeed, GECIs expression can be localized to distinct cellular, even subcellular targets (cytosol, plasma membrane, ER membrane, mitochondria inner or outer membrane, etc.). ${ }^{70}$ Thus, more precise information about compartment-specific calcium dynamics can be obtained compared to highly mobile small-molecule calcium indicators that are usually limited to the cytosol but can sometimes be taken up by organelles ${ }^{87}$ (see Fig. 1). The ubiquitous GECI for detecting calcium signals is the single-fluorophore, intensiometric sensor GCaMP ${ }^{88}$ GCaMP consists of circularly permutated green fluorescent protein (GFP) fused to calmodulin (CaM) via a fragment of myosin light chain kinase (M13). Binding of calcium causes the M13 and CaM domains to interact, limiting proton access to the internal chromophore and producing an increase in fluorescence. Many iterations of improvement have been made to the original GCaMP sensor, ${ }^{88,89}$ with the most widely adopted version being GCaMP6, which has three forms (slow, medium, and fast). GCaMP6 has been reported to outperform synthetic indicator dyes in terms of dynamic range ${ }^{90}$ Continual development of GECIs with different calcium binding affinities $\left(K_{d}\right)$, spectral properties, improved brightness, and signal-to-noise ratios, as well as GECIs that provide effective quantitative measures of calcium concentration, will further expand the ability to study calcium in astrocytes and other cells. Below, we highlight examples of GECI targeting in astrocytes.

The toolkit of GECIs to study calcium transients in astrocytes is becoming increasingly diverse and powerful. This is in part due to the ability to not only target specific cell types but also to target subcellular structures. Initial application of targeted astrocyte GECIs began with Bal Khakh's group in 2013 who compared Lck (membrane-tethered) and cytosolic GCaMP3 ex vivo. The authors found both GECIs allow for effective calcium imaging throughout the entire astrocyte territory (including endfeet), which was an improvement from organic dyes. However, Lck-GCaMP3 provided a higher signal-to-noise ratio for membrane events enabling the detection of small and rapid, possibly synaptic mediated events. ${ }^{91}$ This was corroborated by Stobart et al. who revealed that plasma membrane calcium transients, as reported by Lck-GCaMP6, occur shortly after neuronal events (within $\sim 120 \mathrm{~ms}$ ). Here, the use of targeted GECIs was crucial in the dissociating rapid plasma membrane-based transients from slower onset, $\mathrm{IP}_{3} \mathrm{R}$ mediated, calcium events. ${ }^{39}$ Earlier, Agarwal et al. used an analogous approach where they visualized GFP tethered to mitochondria in astrocytes using a mitochondrial targeting amino acid sequence and combined this with the astrocyte selective expression of GCaMP3 (GLAST-mGC3;mito$E G F) .{ }^{40}$ This allowed them to discover that a significant fraction of astrocyte microdomain calcium signals colocalized with mitochondria. With $\mathrm{IP}_{3}$ signaling and mitochondria activity playing an important role in astrocyte calcium signals, use of intraorganelle GECIs is an exciting avenue for investigation. ${ }^{92}$ Indeed, Okubo recently used G-CEPIA1er, a GECI targeted to the ER in astrocytes, with the sensor facing inside the ER lumen. This unique tool allowed the authors to describe an $\mathrm{IP}_{3} \mathrm{R} 2$-independent signal in $\mathrm{IP}_{3} \mathrm{R} 2-/-$ mice. In response to norepinephrine, which evokes large cytosolic calcium elevations in astrocytes via store calcium release, a significant fraction of the ER calcium drop remained in the absence of $\mathrm{IP}_{3} \mathrm{R} 2 .{ }^{93}$ Recently, Haidey et al. performed spatiotemporal analysis of the endfoot calcium signals in brain slices measured via membrane anchored GCaMP6f. This Lck-GCaMP6f provided high signal-to-noise measures of calcium events localized to the endfoot-vascular membrane. They found basal endfoot calcium transients became more frequent and larger in response to vasoconstriction. ${ }^{61}$ In comparison, using patch loaded Fluo-4 revealed a plateau-like calcium elevation in astrocyte endfeet to the same vasoconstriction (see Fig. 1). This clearly showed how different calcium indicators report the same calcium event differently. The use of subcellular targeted GECIs in astrocytes is still in its infancy, meaning there are many opportunities for discovery. Further work investigating calcium signals from different compartments of the mitochondria in particular could reveal important insights linking metabolism and the ability of astrocytes to feed synapses or control local blood flow.

\section{Imaging Calcium Dynamics in Astrocytes}

Despite dramatic improvements in calcium probes, the experimental results obtained remain constrained by the imaging modalities used. For in vivo imaging, two-photon laser scanning 
microscopy (2PLSM) has become the preferred tool, as good resolution can be acquired at substantial tissue depths $(>500 \mu \mathrm{m}){ }^{94,95}$ 2PLSM requires simultaneous excitation of fluorophores by two long-wavelength photons, which are less susceptible to scattering throughout the tissue. This allows for increased penetration depth, reduced photobleaching, and the photons detected come from fluorophore emission exclusively at the focal plane. ${ }^{96}$ Laser scanning confocal microscopy (LSCM), which employs single-photon short wavelength excitation, is more sensitive to scattering and thus is better suited to imaging more superficial regions (maximum $\sim 50 \mu \mathrm{m}$ from the surface).$^{97}$ This approach utilizes a pinhole to reject fluorescence from out-of-plane sources to improve the resolution of images. Despite both methods being limited by the diffraction of light, the use of shorter wavelengths in LSCM results in superior resolution to 2PLSM as the point spread function or pattern of diffracted light from a point source is smaller (LSCM: $\sim 180 \mathrm{~nm}$ in $x y$ axis; $\sim 500 \mathrm{~nm}$ in $z$ axis versus 2PLSM: $\sim 385 \mathrm{~nm}$ in $x y$ axis; $\sim 1500 \mathrm{~nm}$ in $z$ axis). ${ }^{98-101}$ Given a relatively small point spread function, decreased susceptibility to light scattering and less tissue damage, 2PLSM has been a tool of choice for high resolution fluorescence imaging in vivo, including astrocyte work, whereas LSCM can more easily be employed for live acute slice work. However, 2PLSM is still more commonly utilized in the ex vivo preparation too (see Fig. 2). Using 2PLSM in vivo has revealed new information regarding the effects of different neurotransmitters on microdomain astrocyte calcium transients, where glutamate caused sparse and localized increases in transients, and noradrenaline caused a cellwide elevation. ${ }^{38,40}$

Stimulation emission depletion (STED) microscopy is a powerful super-resolution imaging tool that strikes an appealing compromise between spatial resolution, image acquisition speed, depth, and signal-to-noise ratio, making it particularly well suited to image the fine processes of astrocytes in living tissue. ${ }^{102,103}$ Similar to LSCM and 2PLSM, STED is also a laser scanning method; however, it is not constrained by the diffraction limit of light. ${ }^{104}$ This is accomplished by the addition of a second, redshifted, doughnut-shaped laser that precisely encircles the regular excitation laser, thereby depleting the fluorescent molecules everywhere except the central region of the excitation point spread function. This can also be used in the $z$-axis resulting in super-resolution in all three axes $\left(50 \mathrm{~nm}\right.$ in $x y$ axis; $250 \mathrm{~nm}$ in $z$ axis) ${ }^{105}$ (see Fig. 2). STED microscopy can be used when labeling of the interstitial fluid using a membraneimpermeable dye in a method known as super-resolution shadow imaging (SUSHI). Upon image acquisition, all cellular structures cast a dark "shadow" and instead the extracellular space is illuminated in super-resolution. SUSHI can be combined with a positive fluorescent label of a different color in a specific cell or cell-type of interest, meaning anatomical relationships within the extracellular space can be compared in great detail. ${ }^{105}$

STED microscopy has provided fascinating insights into the nanostructure of the astrocyte arbor. A study by Arizono et al. ${ }^{26}$ found using 3D-STED in combination with confocal calcium imaging that many fine processes connect to form loops near synapses. As such, the morphology of the astrocyte arbor is more similar to a sponge that covers a given territory rather than simply linear processes extending from the soma (see Fig. 2). Further analysis of the spatial relationship between PAPs and excitatory synapses revealed that most localized calcium transients occur in compartmentalized nodes ( 0.07 to $\left.0.7 \mu \mathrm{m}^{2}\right)$, some of which contain organelles. ${ }^{103}$ Correlations between PAP and dendritic spine size, as well as between calcium transient area and spine size were reported suggesting a structural and functional link between PAPs and synapses. ${ }^{26}$ Similarly, it was observed using STED that postsynaptic densities and nodes of Ranvier in the spinal cord associated with astrocyte processes (forming a tripartite synapse) are more structurally complex and contain more diverse arrays of molecular machinery (signaling enzymes, neurotransmitter receptors, etc.). ${ }^{106,107}$

Several groups are currently examining how these microstructures are remodeled under changes in physiological state. As previously mentioned, PAPs withdraw from synapses that have recently undergone LTP induction, which promotes glutamate spillover to extra-synaptic sites. ${ }^{65}$ Increased neuronal activity has also been shown to drive increased expression of gap junction forming protein, connexin 30 (Cx30), on the membrane of PAPs as visualized via STED. ${ }^{108}$ Upregulation of $\mathrm{Cx} 30$ is thought to initiate morphological changes in the astrocyte thereby promoting enhanced glutamate uptake. ${ }^{108,109}$ Recently, SUSHI imaging revealed these loop like PAPs enclose dendrites and interstitial fluid creating a secluded microenvironment. ${ }^{110}$ 


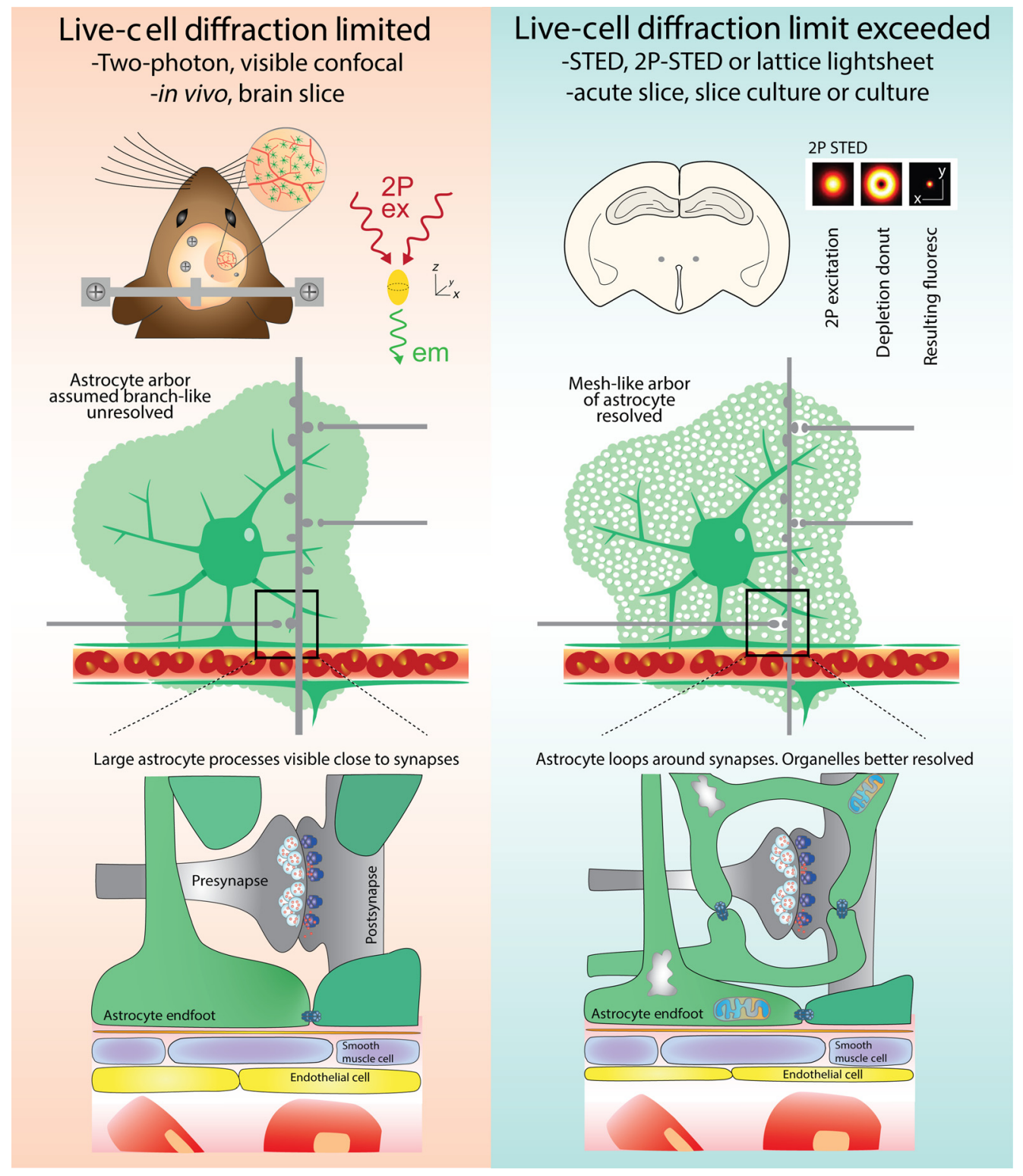

Fig. 2 A schematic comparison of the neuro-glio-vascular interface as observed under diffraction-limited versus diffraction-limit exceeded microscopy for live-cell imaging. Diffraction limited tools (two-photon, visible confocal) are well suited to in vivo and brain slice preparations; however, only large astrocyte processes near synapses are resolved. Diffraction-limit exceeded microscopy (STED, 2P-STED, etc.) in acute slices or culture allow for specific compartments or organelles in addition to the loop-like/spongiform astrocyte arbor to be visualized.

After applying an osmotic stress to the tissue, PAPs and dendrites swell significantly, increasing the contact of these structures while decreasing the volume of interstitial fluid found in the extracellular space. Kaminski's group has examined the link between cytoskeletal (actin/ microtubule) organization on membrane properties using STED and atomic force microscopy in migrating astrocytes. ${ }^{111}$ However, this has not been performed in PAPs undergoing structural changes in response to physiologically relevant stimuli. Investigating cytoskeletal modifications and the influence on membrane properties in this context poses an exciting area of possible study likely contributing to neuro-glia communication.

Although there are numerous exciting applications for STED microscopy, it is important to be cognizant of limitations. While STED provides remarkable super-resolution images of live cell morphology, it is not well suited to capturing astrocyte calcium transients. This can be 
overcome by mapping confocal calcium images onto STED structural images of PAPs. ${ }^{26}$ Another consideration for imaging is that the intensity of the STED (depletion) laser is up to three orders of magnitude greater than the typical excitation laser; therefore, phototoxicity and bleaching are serious concerns. ${ }^{103}$ Several studies have mitigated this issue while still effectively labeling PAPs by patch loading a bright, stable dye (Alexa Fluor 488) directly ${ }^{12}$ or via robust transgenic expression of fluorescent protein ZsGreen. ${ }^{26}$ Image acquisition parameters (laser power, frame rate, and field of view) are important to optimize to further manage phototoxicity and bleaching. ${ }^{103}$ Lastly, the majority of recent STED studies are performed using organotypic slices, which have many advantages in terms of image acquisition/quality. However, this tissue preparation approach is limited if interested in understanding in vivo phenomena or the neurovascular interface. Without blood perfusion, the vascular system collapses; therefore, it is imperative to consider in which contexts STED imaging with organotypic tissue preparation is best applied.

Another interesting optical imaging method that may have utility in capturing ultrafast calcium transients is light sheet fluorescence microscopy (LSFM). A light sheet of micron-scale thickness can be generated from continuous wave lasers. ${ }^{113,114}$ Aligning the light sheet orthogonally to the focal plane of an objective lens enables scanless wide-field optical sectioning and ultrafast image acquisition. ${ }^{15-117}$ Compared to LSFM, conventional point to point scanning methods (LSCM or 2PLSM) are limited in image acquisition speed. ${ }^{118} \mathrm{~A}$ recent study by Pham et al. demonstrated LSFM as useful tool for mapping calcium microdomains in acute brain slices and interestingly, uncovered heterogeneity in the kinetics of spontaneous calcium transients found in cortical versus hippocampal astrocytes. ${ }^{118}$ Despite the speed advantages of this method, the contrast and confocality of LSFM in brain slices appears inferior to two-photon, but there may be room for improvements in sample preparation/presentation within the microscope. Furthermore, LSFM is still a diffraction-limited approach therefore it is imperative to tailor the imaging tool used to ensure it matches the biological question of interest. Nevertheless, more recent lattice lightsheet microscopy breaks the diffraction barrier ${ }^{119}$ and may be a preferred tool moving forward for high speed, super-resolution live-cell work in relatively transparent tissues/organisms. ${ }^{119-121}$

\section{Analysis Tools for Calcium Imaging Data}

Analyzing calcium transients, especially in the astrocyte arbor, is a particularly complex task. These events are numerous and dynamic in time and space, meaning they are not well suited to conventional image analysis methods. Two main strategies to analyze astrocyte calcium imaging data have emerged: (1) region of interest (ROI)-based analysis where calcium transients are treated as individual units in space and (2) event-based analysis where calcium transients are recognized as distinct events in time that are not spatially restricted. When determining which of these approaches are most effective, one should consider the biological question of interest and what information the image analysis is able to provide.

An early attempt to improve the analysis of astrocyte microdomain calcium came from Srinivasan et al., which published the open-source software GECIquant in 2015. GECIquant detects, in a semiautomated fashion, ROIs containing calcium changes above a threshold value. ${ }^{38}$ The software differentiates between somatic calcium fluctuations, waves moving through processes, and punctate signals in microdomains. The program provides the raw fluorescence data over time from each of the detected ROIs. Traces obtained through GECIquant can be further processed via a separate MATLAB script to obtain additional features such as the amplitude and frequency for each ROI detected. ${ }^{48}$

Agarwal et al. developed CaSCaDe, which uses machine-learning to identify calcium events. Thresholding values are used to isolate microdomains that can be further refined based on additional criteria of amplitude and duration. Active regions are plotted onto a spatial map and relevant descriptors (frequency, amplitude, and timing of events) can be extracted. ${ }^{40}$

The development of the program AQuA in 2019 by Poskanzer's group provided additional improvements for astrocyte calcium analysis. AQuA is a departure from ROI-based analysis in favor of an event-based approach. The framework of the ROI is limiting in the context of calcium 


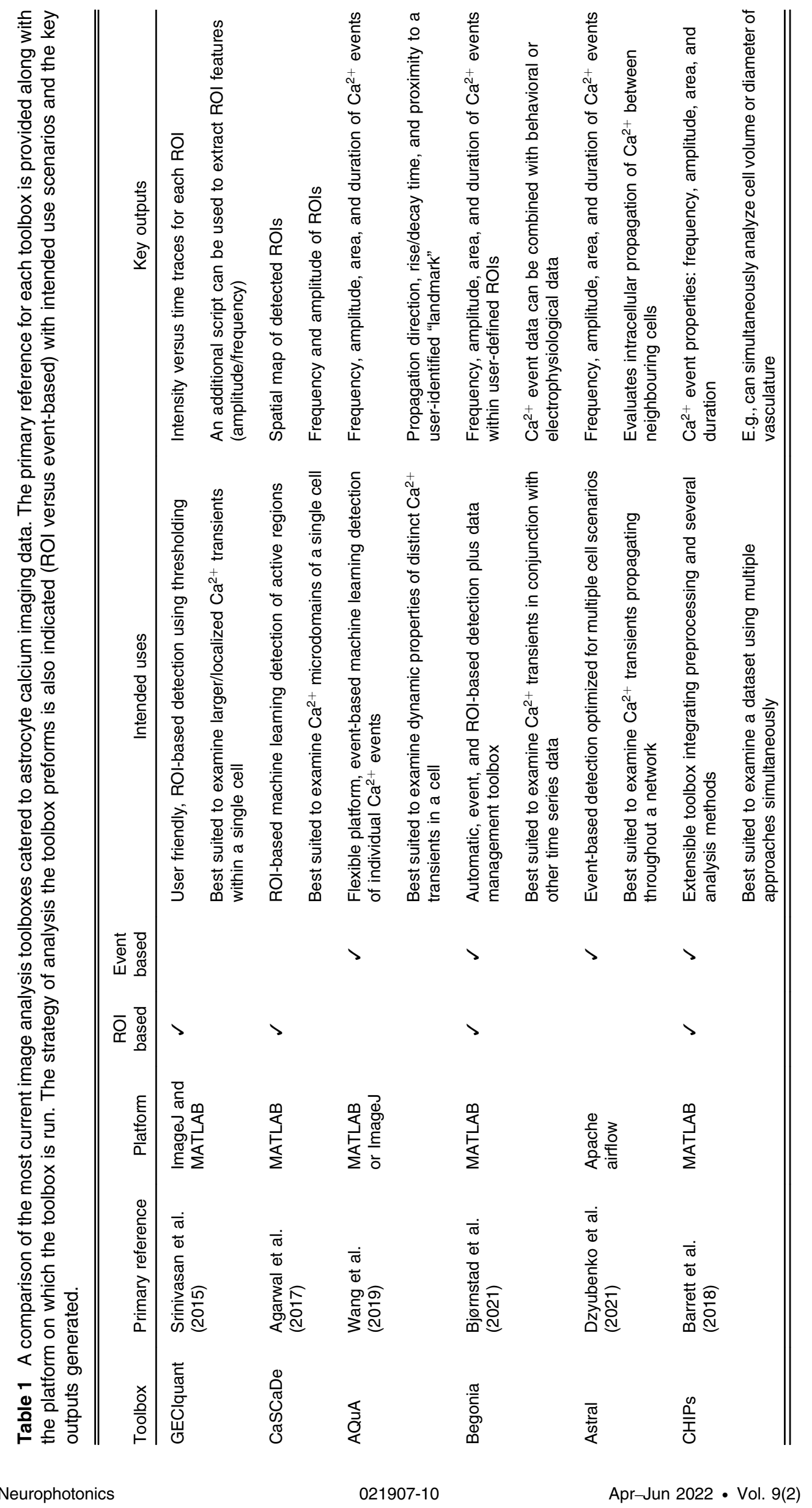


dynamics as there is an assumption that the ROI does not change shape, position, or overlap with other ROIs. ${ }^{122}$ In AQuA, each increase in fluorescent intensity is captured as a unique event that can be analyzed for dynamic properties such as size, shape, propagation direction, duration, frequency, and amplitude. The distinct events are identified through a variety of processing steps, including thresholding, smoothing, and the definition of spatial and temporal boundaries. Throughout the analysis process, the user can inspect traces and modify parameters to ensure optimal analysis. A valuable feature is the ability to choose between different data type presets, depending on the calcium indicator used and on the acquisition signal-to-noise ratio. ${ }^{122}$ Haidey et al. applied AQuA analysis of astrocyte endfoot calcium dynamics in brain slices, to reveal that vasoconstriction did not evoke a new type of calcium transient not previously present, but instead intensified existing spontaneous calcium transients as vessel diameter decreased. ${ }^{61}$ AQuA has also been used to analyze spontaneous astrocyte calcium signaling in Drosophila by the Freeman group. AQuA helped uncover a distinctive subset of spontaneous transients that are activated by local ROS production acting on TRP channel TrmPL, ultimately resulting in changes in oxygen delivery. ${ }^{122,123}$

Integrating complex calcium imaging with electrophysiological or behavioral datasets is a challenge that is addressed by MATLAB toolbox Begonia developed by Bjørnstad et al.. Similar to AQuA, Begonia uses an event-based approach (known as regions of activity or ROAs) to automatically detect calcium signals; however, hand-drawn ROIs can also be incorporated. Contrary to AQuA, however, Begonia allows for easy integration of other time-series data and is optimized for handling large, high-frame-rate $(\sim 30 \mathrm{~Hz})$ datasets, thereby avoiding significantly long processing times. ${ }^{124}$

Majority of the aforementioned analysis packages are targeted to single cell analysis. Software package Astral is designed to analyze astrocyte-astrocyte or astrocyte-neuron interactions at a network level. Astral uses an event based, thresholding method to not only measure parameters of single calcium events (duration, direction, etc.) but also evaluates intercellular propagation of calcium events between neighbors in astrocytic networks. ${ }^{125}$

Another useful image analysis package that has been tailored to suit astrocyte calcium imaging datasets is known as CHIPs, which is a MATLAB-based, open-source toolbox that offers the ability to combine multiple analysis approaches into a single pipeline. ${ }^{26}$ The flexible framework of CHIPs is ideal for examining a dataset in a variety of ways and for developers who are looking to further customize the analysis process. See Table 1 for a comparison of all previously mentioned astrocyte image analysis toolboxes.

\section{Concluding Remarks}

The investigation of astrocytes and their complicated calcium dynamics provides a rich and intriguing area of study in the neurosciences that is undergoing constant development. Our fundamental understanding of the brain has been revolutionized as we continue to recognize the diverse functions of these cells. An overwhelming degree of complexity has emerged, particularly with regard to how astrocytes use calcium signals to orchestrate their many influences on neurons, the vasculature, and other cells. Improved experimental strategies, coupled with the technological advances described here, will provide scientists with the necessary tools to help elucidate the important role of these fascinating cells in neurophysiology.

\section{Disclosures}

The authors declare no competing interests.

\section{Acknowledgments}

This work was supported by the University of Calgary, the Hotchkiss Brain Institute, and Canadian Institutes of Health Research (PJT-173468). G.R.G. was supported by Canada Research Chairs. K.A.G was supported by the Hotchkiss Brain Institute. 


\section{References}

1. A. Araque et al., "Gliotransmitters travel in time and space," Neuron 81, 728-739 (2014).

2. P. Bezzi and A. Volterra, "A neuron-glia signalling network in the active brain," Curr. Opin. Neurobiol. 11, 387-394 (2001).

3. A. Verkhratsky and M. Nedergaard, "Physiology of astroglia," Physiol. Rev. 98, 239-389 (2018).

4. S. Guerra-Gomes et al., "Functional roles of astrocyte calcium elevations: from synapses to behavior," Front. Cell. Neurosci. 11, 1-7 (2018).

5. A. Volterra, N. Liaudet, and I. Savtchouk, "Astrocyte $\mathrm{Ca}^{2+}$ signalling: an unexpected complexity," Nat. Rev. Neurosci. 15, 327-335 (2014).

6. E. A. Bushong et al., "Protoplasmic astrocytes in CA1 stratum radiatum occupy separate anatomical domains," J. Neurosci. 22, 183-192 (2002).

7. E. Bindocci et al., "Neuroscience: three-dimensional $\mathrm{Ca}^{2+}$ imaging advances understanding of astrocyte biology," Science 356 (2017).

8. J. P. Heller and D. A. Rusakov, "The nanoworld of the tripartite synapse: insights from super-resolution microscopy," Front. Cell. Neurosci. 11, 1-14 (2017).

9. M. Usowicz, V. Gallo, and S. Cull-Candy, "Multiple conductance channels in type-2 cerebellar astrocytes activated by excitatory amino acids," Nature 339(6223), 380-383 (1989).

10. A. Cornell-Bell et al., "Glutamate induces calcium waves in cultured astrocytes: longrange glial signaling," Science 247(4941), 470-473 (1990).

11. J. W. Dani, A. Chernjavsky, and S. J. Smith, "Neuronal activity triggers calcium waves in hippocampal astrocyte networks," Neuron 8, 429-440 (1992).

12. E. A. Newman and K. R. Zahs, "Modulation of neuronal activity by glial cells in the retina," J. Neurosci. 18, 4022-4028 (1998).

13. X. Wang et al., "Astrocytic $\mathrm{Ca}^{2+}$ signaling evoked by sensory stimulation in vivo," Nat. Neurosci. 9, 816-823 (2006).

14. S. Coco et al., "Storage and release of ATP from astrocytes in culture," J. Biol. Chem. 278, 1354-1362 (2003).

15. L. Pellerin and P. J. Magistretti, "Glutamate uptake into astrocytes stimulates aerobic glycolysis: a mechanism coupling neuronal activity to glucose utilization," Proc. Natl. Acad. Sci. U. S. A. 91, 10625-10629 (1994).

16. M. Krzan et al., "Calcium-dependent exocytosis of atrial natriuretic peptide from astrocytes," J. Neurosci. 23, 1580-1583 (2003).

17. M. A. Di Castro et al., "Local $\mathrm{Ca}^{2+}$ detection and modulation of synaptic release by astrocytes," Nat. Neurosci. 14, 1276-1284 (2011).

18. C. Henneberger et al., "Long-term potentiation depends on release of d-serine from astrocytes," Nature 463, 232-236 (2010).

19. M. Zonta et al., "Neuron-to-astrocyte signaling is central to the dynamic control of brain microcirculation," Nat. Neurosci. 6, 43-50 (2003).

20. T. Takano et al., "Astrocyte-mediated control of cerebral blood flow," Nat. Neurosci. 9, 260-267 (2006).

21. S. J. Mulligan and B. A. MacVicar, "Calcium transients in astrocyte endfeet cause cerebrovascular constrictions," Nature 431, 195-199 (2004).

22. G. J. Gordon, S. J. Mulligan, and B. A. MacVicar, "Astrocyte control of the cerebrovasculature," Glia 55, 1214-1221 (2007).

23. D. A. Rusakov, "Disentangling calcium-driven astrocyte physiology," Nat. Rev. Neurosci. 16, 226-233 (2015).

24. J. Grosche et al., "Microdomains for neuron-glia interaction: parallel fiber signaling to Bergmann glial cells," Nat. Neurosci. 2, 139-143 (1999).

25. A. Semyanov and A. Verkhratsky, "Astrocytic processes: from tripartite synapses to the active milieu," Trends Neurosci. 44, 781-792 (2021).

26. M. Arizono et al., "Structural basis of astrocytic $\mathrm{Ca}^{2+}$ signals at tripartite synapses," Nat. Commun. 11, 1-15 (2020). 
27. K. Watanabe et al., "Three-dimensional organization of the perivascular glial limiting membrane and its relationship with the vasculature: a scanning electron microscope study," Okajimas Folia Anat. Jpn. 87, 109-121 (2010).

28. R. Ventura and K. M. Harris, "Three-dimensional relationships between hippocampal synapses and astrocytes," J. Neurosci. 19, 6897-6906 (1999).

29. K. P. Lehre and D. A. Rusakov, "Asymmetry of glia near central synapses favors presynaptically directed glutamate escape," Biophys. J. 83, 125-134 (2002).

30. M. R. Witcher et al., "Three-dimensional relationships between perisynaptic astroglia and human hippocampal synapses," Glia 58, 572-587 (2010).

31. I. Patrushev et al., "Subcellular location of astrocytic calcium stores favors extrasynaptic neuron-astrocyte communication," Cell Calcium 54, 343-349 (2013).

32. T. M. Mathiisen et al., "The perivascular astroglial sheath provides a complete covering of the brain microvessels: an electron microscopic 3D reconstruction," Glia 58, 1094-1103 (2010).

33. C. Calì et al., "3D cellular reconstruction of cortical glia and parenchymal morphometric analysis from Serial Block-Face electron microscopy of juvenile rat," Prog. Neurobiol. 183, 101696 (2019).

34. A. Aboufares El Alaoui et al., "Characterization of subcellular organelles in cortical perisynaptic astrocytes," Front. Cell. Neurosci. 14, 573944 (2020).

35. L. A. Holtzclaw et al., "Astrocytes in adult rat brain express type 2 inositol 1,4,5trisphosphate receptors," Glia 39, 69-84 (2002).

36. J. Petravicz, K. M. Boyt, and K. D. McCarthy, "Astrocyte $\mathrm{IP}_{3} \mathrm{R} 2$-dependent $\mathrm{Ca}^{2+}$ signaling is not a major modulator of neuronal pathways governing behavior," Front. Behav. Neurosci. 8, 384 (2014).

37. N. Takata et al., "Astrocyte calcium signaling transforms cholinergic modulation to cortical plasticity in vivo," J. Neurosci. 31, 18155-18165 (2011).

38. R. Srinivasan et al., " $\mathrm{Ca}^{2+}$ signaling in astrocytes from $\operatorname{Ip} 3 r 2(-/-)$ mice in brain slices and during startle responses in vivo," Nat. Neurosci. 18, 708-717 (2015).

39. J. L. Stobart et al., "Cortical circuit activity evokes rapid astrocyte calcium signals on a similar timescale to neurons," Neuron 98, 726-735.e4 (2018).

40. A. Agarwal et al., "Transient opening of the mitochondrial permeability transition pore induces microdomain calcium transients in astrocyte processes," Neuron 93, 587-605.e7 (2017).

41. S. V. Straub et al., "Dynamic inositol trisphosphate-mediated calcium signals within astrocytic endfeet underlie vasodilation of cerebral arterioles," J. Gen. Physiol. 128, 659-669 (2006).

42. S. J. Bradley and R. A. J. Challiss, "G protein-coupled receptor signalling in astrocytes in health and disease: a focus on metabotropic glutamate receptors," Biochem. Pharmacol. 84, 249-259 (2012).

43. T. A. Fiacco and K. D. McCarthy, "Intracellular astrocyte calcium waves in situ increase the frequency of spontaneous AMPA receptor currents in CA1 pyramidal neurons," J. Neurosci. 24, 722-732 (2004).

44. W. Sun et al., "Glutamate-dependent neuroglial calcium signaling differs between young and adult brain," Science 339, 197-200 (2013).

45. M. Lavialle et al., "Structural plasticity of perisynaptic astrocyte processes involves ezrin and metabotropic glutamate receptors," Proc. Natl. Acad. Sci. U. S. A. 108, 12915-12919 (2011).

46. M. Arizono et al., "Receptor-selective diffusion barrier enhances sensitivity of astrocytic processes to metabotropic glutamate receptor stimulation," Sci. Signal. 5, ra27 (2012).

47. A. Serrano et al., "GABAergic network activation of glial cells underlies hippocampal heterosynaptic depression," J. Neurosci. 26, 5370-5382 (2006).

48. L. Mariotti et al., "Interneuron-specific signaling evokes distinctive somatostatin-mediated responses in adult cortical astrocytes," Nat. Commun. 9, 82 (2018).

49. C. A. Durkee et al., "G(i/o) protein-coupled receptors inhibit neurons but activate astrocytes and stimulate gliotransmission," Glia 67, 1076-1093 (2019). 
50. M. W. Sherwood et al., "Astrocytic $\mathrm{IP}_{3}$ Rs: beyond $\mathrm{IP}_{3} \mathrm{R} 2$," Front. Cell. Neurosci. 15, 282 (2021).

51. J. Wahis and M. G. Holt, "Astrocytes, noradrenaline, $\alpha 1$-adrenoreceptors, and neuromodulation: evidence and unanswered questions," Front. Cell. Neurosci. 15 (2021).

52. R. L. Rungta et al., " $\mathrm{Ca}^{2+}$ transients in astrocyte fine processes occur via $\mathrm{Ca}^{2+}$ influx in the adult mouse hippocampus," Glia 64, 2093-2103 (2016).

53. A. Minelli et al., "GAT-3, a high-affinity GABA plasma membrane transporter, is localized to astrocytic processes, and it is not confined to the vicinity of GABAergic synapses in the cerebral cortex," J. Neurosci. 16, 6255-6264 (1996).

54. N. C. Danbolt, J. Storm-Mathisen, and B. I. Kanner, "An [Na+ + K+]coupled L-glutamate transporter purified from rat brain is located in glial cell processes," Neuroscience 51, 295-310 (1992).

55. K. Sakers et al., "Astrocytes locally translate transcripts in their peripheral processes," Proc. Natl. Acad. Sci. U. S. A. 114, E3830-E3838 (2017).

56. K. Boddum et al., "Astrocytic GABA transporter activity modulates excitatory neurotransmission," Nat. Commun. 7, 13572 (2016).

57. E. Shigetomi et al., "TRPA1 channels are regulators of astrocyte basal calcium levels and long-term potentiation via constitutive d-serine release," J. Neurosci. 33, 10143-10153 (2013).

58. E. B. Malarkey, Y. Ni, and V. Parpura, " $\mathrm{Ca}^{2+}$ entry through TRPC1 channels contributes to intracellular $\mathrm{Ca}^{2+}$ dynamics and consequent glutamate release from rat astrocytes," Glia 56, 821-835 (2008).

59. N. Marina et al., "Astrocytes monitor cerebral perfusion and control systemic circulation to maintain brain blood flow," Nat. Commun. 11, 131 (2020).

60. K. J. Kim et al., "Astrocyte contributions to flow/pressure-evoked parenchymal arteriole vasoconstriction," J. Neurosci. 35, 8245-8257 (2015).

61. J. N. Haidey et al., "Astrocytes regulate ultra-slow arteriole oscillations via stretchmediated TRPV4-COX-1 feedback," Cell Rep. 36, 109405 (2021).

62. J. M. Beckel et al., "Mechanosensitive release of adenosine 5'-triphosphate through pannexin channels and mechanosensitive upregulation of pannexin channels in optic nerve head astrocytes: a mechanism for purinergic involvement in chronic strain," Glia 62, 1486-1501 (2014).

63. M. Velasco-Estevez et al., "Piezo1 regulates calcium oscillations and cytokine release from astrocytes," Glia 68, 145-160 (2020).

64. E. A. Turovsky et al., "Mechanosensory signaling in astrocytes," J. Neurosci. 40, 9364-9371 (2020).

65. C. Henneberger et al., "LTP induction boosts glutamate spillover by driving withdrawal of perisynaptic astroglia," Neuron 108, 919-936.e11 (2020).

66. Y. Bernardinelli et al., "Activity-dependent structural plasticity of perisynaptic astrocytic domains promotes excitatory synapse stability," Curr. Biol. 24, 1679-1688 (2014).

67. D. Molotkov et al., "Calcium-induced outgrowth of astrocytic peripheral processes requires actin binding by Profilin-1," Cell Calcium. 53, 338-348 (2013).

68. A. Minta, J. P. Kao, and R. Y. Tsien, "Fluorescent indicators for cytosolic calcium based on rhodamine and fluorescein chromophores," J. Biol. Chem. 264, 8171-8178 (1989).

69. G. Grynkiewicz, M. Poenie, and R. Y. Tsien, "A new generation of $\mathrm{Ca}^{2+}$ indicators with greatly improved fluorescence properties," J. Biol. Chem. 260, 3440-3450 (1985).

70. J. T. Lock, I. Parker, and I. F. Smith, "A comparison of fluorescent $\mathrm{Ca}^{2+}$ indicators for imaging local $\mathrm{Ca}^{2+}$ signals in cultured cells," Cell Calcium 58, 638-648 (2015).

71. T. Hendel et al., "Fluorescence changes of genetic calcium indicators and OGB-1 correlated with neural activity and calcium in vivo and in vitro," J. Neurosci. 28, 7399-7411 (2008).

72. S. Ding, "In vivo imaging of $\mathrm{Ca}^{2+}$ signaling in astrocytes using two-photon laser scanning fluorescent microscopy," Methods Mol. Biol. 814, 545-554 (2012).

73. A. M. B. Reeves, E. Shigetomi, and B. S. Khakh, "Bulk loading of calcium indicator dyes to study astrocyte physiology: key limitations and improvements using morphological maps," J. Neurosci. 31, 9353-9358 (2011). 
74. O. Garaschuk, R.-I. Milos, and A. Konnerth, "Targeted bulk-loading of fluorescent indicators for two-photon brain imaging in vivo," Nat. Protoc. 1, 380-386 (2006).

75. Y. Fujii, S. Maekawa, and M. Morita, "Astrocyte calcium waves propagate proximally by gap junction and distally by extracellular diffusion of ATP released from volume-regulated anion channels," Sci. Rep. 7, 13115 (2017).

76. M. K. Herde et al., "Local efficacy of glutamate uptake decreases with synapse size," Cell Rep. 32, 108182 (2020).

77. A. Nimmerjahn et al., "Sulforhodamine 101 as a specific marker of astroglia in the neocortex in vivo," Nat. Methods 1, 31-37 (2004).

78. K. W. Kafitz et al., "Developmental profile and properties of sulforhodamine 101: labeled glial cells in acute brain slices of rat hippocampus," J. Neurosci. Methods 169, 84-92 (2008).

79. C. Schnell, Y. Hagos, and S. Hulsmann, "Active sulforhodamine 101 uptake into hippocampal astrocytes," PLoS One 7, 1-10 (2012).

80. J. Kang et al., "Sulforhodamine 101 induces long-term potentiation of intrinsic excitability and synaptic efficacy in hippocampal CA1 pyramidal neurons," Neuroscience 169, 1601-1609 (2010).

81. O. Garaschuk, "Imaging microcircuit function in healthy and diseased brain," Exp. Neurol. 242, 41-49 (2013).

82. C. Nolte et al., "GFAP promoter-controlled EGFP-expressing transgenic mice: a tool to visualize astrocytes and astrogliosis in living brain tissue," Glia 33, 72-86 (2001).

83. V. Vives et al., "Visualization of S100B-positive neurons and glia in the central nervous system of EGFP transgenic mice," J. Comp. Neurol. 457, 404-419 (2003).

84. N. Heintz, "Gene expression nervous system Atlas (GENSAT)," Nat. Neurosci. 7, 483 (2004).

85. Y. Zuo et al., "Fluorescent proteins expressed in mouse transgenic lines mark subsets of glia, neurons, macrophages, and dendritic cells for vital examination," J. Neurosci. 24, 10999-11009 (2004).

86. M. R. Regan et al., "Variations in promoter activity reveal a differential expression and physiology of glutamate transporters by glia in the developing and mature CNS," J. Neurosci. 27, 6607-6619 (2007).

87. E. Boitier, R. Rea, and M. R. Duchen, "Mitochondria exert a negative feedback on the propagation of intracellular $\mathrm{Ca}^{2+}$ waves in rat cortical astrocytes," J. Cell Biol. 145, 795-808 (1999).

88. J. Nakai, M. Ohkura, and K. Imoto, "A high signal-to-noise $\mathrm{Ca}^{2+}$ probe composed of a single green fluorescent protein," Nat. Biotechnol. 19, 137-141 (2001).

89. G. S. Baird, D. A. Zacharias, and R. Y. Tsien, "Circular permutation and receptor insertion within green fluorescent proteins," Proc. Natl. Acad. Sci. U. S. A. 96, 11241-11246 (1999).

90. T.-W. Chen et al., "Ultrasensitive fluorescent proteins for imaging neuronal activity," Nature 499, 295-300 (2013).

91. E. Shigetomi et al., "Imaging calcium microdomains within entire astrocyte territories and endfeet with GCaMPs expressed using adeno-associated viruses," J. Gen. Physiol. 141, 633-647 (2013).

92. J. Suzuki et al., "Imaging intraorganellar $\mathrm{Ca}^{2+}$ at subcellular resolution using CEPIA," Nat. Commun. 5, 4153 (2014).

93. Y. Okubo et al., "Inositol 1,4,5-trisphosphate receptor type 2-independent $\mathrm{Ca}^{2+}$ release from the endoplasmic reticulum in astrocytes," Glia 67, 113-124 (2019).

94. P. Theer, M. T. Hasan, and W. Denk, "Two-photon imaging to a depth of 1000 microm in living brains by use of a $\mathrm{Ti}: \mathrm{Al}_{2} \mathrm{O}_{3}$ regenerative amplifier," Opt. Lett. 28, 1022-1024 (2003).

95. F. Helmchen and W. Denk, "Deep tissue two-photon microscopy," Nat. Methods 2, 932-940 (2005).

96. K. Svoboda and R. Yasuda, "Principles of two-photon excitation microscopy and its applications to neuroscience," Neuron 50, 823-839 (2006).

97. V. E. Centonze and J. G. White, "Multiphoton excitation provides optical sections from deeper within scattering specimens than confocal imaging," Biophys. J. 75, 2015-2024 (1998). 
98. R. W. Cole, T. Jinadasa, and C. M. Brown, "Measuring and interpreting point spread functions to determine confocal microscope resolution and ensure quality control," Nat. Protoc. 6, 1929-1941 (2011).

99. A. Pérez-Alvarez, A. Araque, and E. Martín, "Confocal microscopy for astrocyte in vivo imaging: recycle and reuse in microscopy," Front. Cell. Neurosci. 7, 51 (2013).

100. D. G. Rosenegger et al., "A high performance, cost-effective, open-source microscope for scanning two-photon microscopy that is modular and readily adaptable," PLoS One 9 (2014).

101. L. Schermelleh, R. Heintzmann, and H. Leonhardt, "A guide to super-resolution fluorescence microscopy," J. Cell Biol. 190, 165-175 (2010).

102. J. Tønnesen and U. V. Nägerl, "Two-color STED imaging of synapses in living brain slices," Methods Mol. Biol. 950, 65-80 (2013).

103. M. Arizono and U. V. Nägerl, "Deciphering the functional nano-anatomy of the tripartite synapse using stimulated emission depletion microscopy," Glia 4, 607-618 (2021).

104. S. Hell, "Far-field optical nanoscopy," Science 316, 1153-1158 (2007).

105. J. Tønnesen, V. V. G. K. Inavalli, and U. V. Nägerl, "Super-resolution imaging of the extracellular space in living brain tissue," Cell 172, 1108-1121.e15 (2018).

106. M. J. Broadhead et al., "Nanostructural diversity of synapses in the mammalian spinal cord," Sci. Rep. 10, 8189 (2020).

107. D. R. Serwanski, P. Jukkola, and A. Nishiyama, "Heterogeneity of astrocyte and NG2 cell insertion at the node of ranvier," J. Comp. Neurol. 525, 535-552 (2017).

108. G. Ghézali et al., "Neuronal activity drives astroglial connexin 30 in perisynaptic processes and shapes its functions," Cereb. Cortex 30, 753-766 (2020).

109. U. Pannasch et al., "Connexin 30 sets synaptic strength by controlling astroglial synapse invasion," Nat. Neurosci. 17, 549-558 (2014).

110. M. Arizono et al., "Super-resolution shadow imaging reveals local remodeling of astrocytic microstructures and brain extracellular space after osmotic challenge," Glia 69, 1605-1613 (2021).

111. N. Curry et al., "Correlative STED and atomic force microscopy on live astrocytes reveals plasticity of cytoskeletal structure and membrane physical properties during polarized migration," Front. Cell. Neurosci. 11, 104 (2017).

112. A. Panatier, M. Arizono, and U. V. Nägerl, "Dissecting tripartite synapses with STED microscopy," Philos. Trans. R. Soc. Lond. Ser. B, Biol. Sci. 369, 20130597 (2014).

113. P. J. Keller and H.-U. Dodt, "Light sheet microscopy of living or cleared specimens," Curr. Opin. Neurobiol. 22, 138-143 (2012).

114. J. G. Ritter et al., "Light sheet microscopy for single molecule tracking in living tissue," PLoS One 5, e11639 (2010).

115. M. B. Ahrens et al., "Whole-brain functional imaging at cellular resolution using lightsheet microscopy," Nat. Methods 10, 413-420 (2013).

116. M. Jemielita et al., "Comparing phototoxicity during the development of a zebrafish craniofacial bone using confocal and light sheet fluorescence microscopy techniques," J. Biophotonics 6, 920-928 (2013).

117. R. M. Power and J. Huisken, "A guide to light-sheet fluorescence microscopy for multiscale imaging," Nat. Methods 14, 360-373 (2017).

118. C. Pham et al., "Mapping astrocyte activity domains by light sheet imaging and spatiotemporal correlation screening," Neuroimage 220, 117069 (2020).

119. A. Stockhausen et al., "Hard-wired lattice light-sheet microscopy for imaging of expanded samples," Opt. Express 28, 15587-15600 (2020).

120. C.-Y. Chen et al., "The applications of lattice light-sheet microscopy for functional volumetric imaging of hippocampal neurons in a three-dimensional culture system," Micromachines 10, 509 (2019).

121. B.-C. Chen et al., "Lattice light-sheet microscopy: imaging molecules to embryos at high spatiotemporal resolution," Science 346, 1257998 (2014).

122. Y. Wang et al., "Accurate quantification of astrocyte and neurotransmitter fluorescence dynamics for single-cell and population-level physiology," Nat. Neurosci. 22, 1936-1944 (2019). 
123. Z. Ma and M. R. Freeman, "TrpML-mediated astrocyte microdomain $\mathrm{Ca}^{2+}$ transients regulate astrocyte-tracheal interactions," Elife 9, e58952 (2020).

124. D. M. Bjørnstad et al., "Begonia-a two-photon imaging analysis pipeline for astrocytic $\mathrm{Ca}^{2+}$ signals," Front. Cell. Neurosci. 15, 681066 (2021).

125. E. Dzyubenko et al., "Analysing intercellular communication in astrocytic networks using 'Astral',' Front. Cell. Neurosci. 15, 689268 (2021).

126. M. J. P. Barrett et al., "CHIPS: an extensible toolbox for cellular and hemodynamic two-photon image analysis," Neuroinformatics 16, 145-147 (2018).

Kelsea A. Gorzo is currently a $\mathrm{PhD}$ student at the University of Calgary's Hotchkiss Brain Institute and the Universitié de Bordeaux Neurocampus. Her research focuses on how astrocyte mechanosensing modulates calcium dynamics, subsequently influencing metabolism and synaptic plasticity. To investigate this, she uses tools such as genetically encoded calcium indicators, two-photon imaging and super resolution, and stimulation emission depletion imaging.

Grant R. Gordon is an associate professor at the University of Calgary and a member of the Hotchkiss Brain Institute. His lab investigates the role astrocytes in neurovascular coupling, synaptic plasticity, and brain metabolism. They aim to discover new mechanisms in health and better understand conditions such as stress, epilepsy, stroke, and dementia. Two-photon fluorescence imaging, electrophysiology and genetically encoded actuators and biosensors are the tools they apply both ex vivo and in vivo. 